\title{
MALARIA VACCINE DEVELOPMENT: PREVIOUS SUCCESS, CURRENT STATUS AND FUTURE PROSPECTS
}

\author{
Yusuf Eshimutu Abu \\ Department of Microbiology, \\ Federal University of Technology, Minna. Nigeria. \\ Yemisi D. Ajala \\ Department of Microbiology, \\ Federal University of Technology, Minna. Nigeria. \\ Patrick Legbo \\ Department of Microbiology \\ Federal University of Technology, Minna. Nigeria.
}

\begin{abstract}
Malaria has led to several cases of death and illness all over the world. About million cases are reported every year. More than $90 \%$ of cases are reported in Sub-Saharan Africa. The goal in the development of highly effective and durable vaccines against the human malaria parasites Plasmodium falciparum and $P$. vivax remains a key priority to the world with great importance. This review is a case study on the development of Malaria Vaccine from the previous success through to current status and future prospect.
\end{abstract}

Keywords: Cases, Development, Malaria, Plasmodium, Vaccine.

\section{INTODUCTION}

Malaria has been reported as one of the deadliest disease that affect people of all ages (WHO, 2018). It is more endemic and deadly in Africa compared to other regions (Rénia, 2018). Malaria is caused by five species of Plasmodium that infect humans (Plasmodium falciparum, Plasmodium vivax, Plasmodium ovale spp., Plasmodium malariae and Plasmodium knowlesi) and is transmitted by the bite of infected female Anopheline mosquitoes (Birkett, 2016). World Malaria Report released in November 2018, revealed an increased in malaria cases from 217 million cases in 2016 to 219 million cases of malaria in 2017. The estimated number of malaria deaths stood at 435000 in
Hajarah Muhammad Bashar

Department of Microbiology, Federal University of Technology, Minna. Nigeria

Chizoba Eze

Department of Microbiology, Federal University of Technology, Minna. Nigeria

Adeniyi Kamoru Abdulazeez

Department of Animal Biology,

Federal University of Technology, Minna. Nigeria

2017 according to World Health Organization Report, 2019.

In 2010, approximately 3.3 billion people were at risk of malaria (Birkett, et al., 2013). About half of the world population was at risk with the highest burden in Sub-Sahara Africa. Most malaria cases and deaths occur in sub-Saharan Africa. However, the WHO regions of South-East Asia, Eastern Mediterranean, Western Pacific, and the Americas are also at risk (Rénia, 2018).

Risk levels and severity of malaria depends on age (infants, children under 5 years of age), condition (pregnant women, non-immune migrants, mobile populations and travelers) and immune status (patient living with HIV/AIDS) (WHO, 2018).

Over the past few years, malaria burden has reduced. In spite of this remarkable achievements, some countries in Africa are left out as some still show stable (moderate-to-high) malaria transmission and are without any good reduction in malaria disease (WHO, 2014). The mutating nature of the Plasmodium species makes it necessary to control malaria with all effective methods simultaneously. Recently, parasite resistance to artemisinin has been detected in some countries in Southeast Asia (Ashley et al., 2014). This could result to a possible spread to other regions of the world. Even Although it is capital-intensive to use all effective control measures, the investments are likely to 
eventually impact significantly on malaria burden. According to Sachs and Malaney (2002), the yearly cost of malaria to sub-Saharan Africa is approximately US $\$ 12$ billion, and it is projected that this may slow up economic growth by up to $1.3 \%$ every year.

It is no longer news that vaccines have been shown to significantly reduce the burden of several viral and bacterial diseases. Malaria vaccines has the potential to effectively reduce the incidence of malaria. So far, the RTS,S malaria vaccine is the only approved vaccine against malaria under the trade name of Mosquirix (Olotu et al., 2016).

According to Asante et al (2016), there is broad agreement that development of new and effective vaccine products cannot proceed without a detailed understanding of the immune correlates of protection. This is imperative in the design and evaluation of vaccine efficacy. Gathering such data is usually dependent upon having material from patients, for example those who have recovered from a natural infection, and the availability of an animal model which responds to the infection and the vaccine formulation in a very similar manner to humans. Where these are lacking, vaccine development is considerably more difficult, and this has too often led to failure of clinical trials results or even serious safety concerns (Oyston and Robinson, 2012).

A comprehensive and detailed approach have been employed in malaria vaccine development which is dependent on multiple epidemiological and experimental studies. Individuals living in endemic regions acquire immunity over time after repeated exposure to the parasites. Such immunity, also called premonition, is partial, species-specific and biphasic. At the initial stage, the hosts still get infected but do not develop clinical symptoms. Conversely, subsequent exposure, prevent or limit parasite multiplication, it takes long to time to develop. This second phase is heavily dependent on parasite exposure - increased exposure of the host is inversely proportioanal to the development of immunity. Hence, the goal of vaccine strategies is to reduce the time needed to acquire protective immunity and to make the immunity longlasting (Rénia et al., 2018).

\section{RATIONALE AND GOALS FOR MALARIA VACCINE DEVELOPMENT}

Over the years, evidence has shown that people become resistant to severe, life-threatening and then to clinical disease due to repeated exposure to the parasite especially with people born in endemic areas who survive first year of exposure (Doolan et al., 2009). Frequent re-exposure is required to maintain this condition of immunity with infection (concomitant immunity). Transfer of gamma-globulin fractions from semi-immune to susceptible humans mitigates malaria disease demonstrating that clinical protection from malaria is possible, and that immunoglobulin targeting malaria antigens can play a critical role (Cohen et al., 1961; Bouharoun-Tayoun et al., 1990). Inoculation of humans with irradiated sporozoites by mosquito bite can prevent the emergence of blood-stage infection after subsequent experimental challenge demonstrating the possibility of inducing high level protection against infection under experimental conditions (Clyde, et al 1973; Cochrane, et al., 1980).

The primary refinement introduced by the malaria eradication R\&D agenda setting process during 20092010 is the confirmation that for elimination and global eradication, impact on transmission rather than morbidity is the paramount efficacy outcome. There is general agreement that malaria eradication is not possible with the currently available tools. Development of a highly efficacious malaria vaccine which dramatically reduces transmission would be a transformative tool that could enable future eradication (Schwartz et al., 2012).

\section{PREVIOUS SUCCESS ON MALARIA VACCINE DEVELOPMENT}

Early malaria vaccine research began in the 1930s aim at inactivating or killing parasites that failed to generate a protective immune response. The addition of adjuvant systems demonstrated immunogenicity of malaria vaccine candidates in animal models; Jules Freund and colleagues demonstrated partial protection in ducklings (Freund et al., 1945). Subsequent vaccine development efforts used rodent malaria models. This led to the first human malaria vaccine trial with demonstrated efficacy, a study that delivered irradiated P. falciparum sporozoites to vaccinees by mosquito bite (Clyde et al., 1973).

There have been significant decrease in malaria mortality in the last $10-15$ years, and the most advanced pre-erythrocytic malaria vaccine, RTS,S received a positive opinion from European regulators in July 2015 (Doolan et al., 2009). However, no blood stage vaccine has reached a phase III trial, clinical investigation is still being carried out. Some of the vaccine under clinical development are; 
EBA175: A cysteine-rich second region of (EBA-175RII) vaccine has been tested in a phase Ia trial, and the vaccine induced GIA - active antibodies. In a recent rabbit study, antibodies against more conserved regions of EBA-175 (region III-V) showed stronger and more strain-transcending activities judged by GIA (Healer $e t$ al., 2013), while the functional activity of regions III-V is unknown. Further investigation is required to reveal whether the region III-V vaccine is better than the RII vaccine in humans since different field parasite show different protein expression levels of EBA and $\mathrm{Rh}$ proteins (EBA-140, EBA-175, EBA-181, RH1, and RH2 were tested in the study) (Bei et al., 2007), all of which are involved in redundant merozoite invasion pathways, a combination with other antigen(s) is likely to be required to show efficacy in the field.

P27A: This is a part of Trophozoite exported protein 1 (Tex 1, previously called hypothetical protein PFF0165C). The Tex 1 antigen was found by a unique approach, that is based on the a-helical coiled coil structure of the molecule (Agak et al., 2007). In contrast to other blood-stage candidates, tex 1 is not a merozite protein, and locates at Maurer's clefts in infected erythrocytes (Dietz O, et al. 2012). Human affinity-purified P27A - specific antibody and rabbit anti-P27A anti body showed ADCI activities (Dietz et al., 2012; Bang et al., 2009), and the first phase 1a and $1 \mathrm{~b}$ trial with Alhydrogel or GLA -SE adjuvants was completed in July 2015 (Clinical Trials. gov identifier NCTO1949909). Several other antigens tested in the structure - based screening study showed ADCI activities (Agak et al., 2007), and they are also interesting candidates for further investigation.

RH5: This is one of the reticulocyte binding-like homologue (Rh) proteins, and an RH5 vaccine which could induce strain-transcending antibodies in animals judged by GIA (Douglas et al., 2011). In addition, while the antibody levels in malaria-exposed individuals are low compared to other merozoite antigens (Douglas et al., 2011; Coursen et al., 2014), affinity-purified human anti-RH5 IgGs - also showed GIA activity (Ahouidi, et al., 2013; Coursen et al., 2014). The low immunogenicity in humans may explain the reason why there are very limited polymorphisms in the molecule (Bartholdson et al., 2013). In an Aotus monkey study where the monkeys were immunized with the 3D7 sequence of RH5 using human-compatible vaccine formulations (chAd63 vaccination followed by either MVA boost, or recombinant RH5 protein-Abisco - 100 adjuvant boost), the vaccine induced a protective effect against heterologus FVO parasite challenge (Baldeviano et al., 2015). A phase 1a trial with chAd63 - MVA vaccines is ongoing (Clinical Trials. gov identifier NCTO2181088). In addition to studies with RH5 as a stand alone vaccine candidates, many animal immunization studies have been conducted using mixtures of vaccines including RH5. For example, P.falciparum RH5 interacting protein (PfRipr) (Chen et al., 2011) and cysteine - rich protective antigen (Amlabu et al., 2015), both of which make a complex with RH5 during the merozoite invasion, can induce GIA-active antibodies by themselves and in combination with RH5. Furthermore, other merozite antigens, such as EBA - 175, RH1, RH2, RH4, AARP (apical asparagines-rich protein) and PF 38, have also been evaluated with RH5 (Chen et al., 2011; Douglas et al., 2012; Ahouidi et al., 2013), and the vaccines cross-reactive functional antibodies judged by GIA of interest, some combinations of IgGs showed synergistic invasion inhibition in GIA (Amlabu et al., 2015; Douglas et al., 2015). At these moment, it is not clear whether such synergistic protective effects can be observed in vivo.

A future sporozoit vaccine would be expected to include the NANP epitope, a specific $T$ cell epitope (which may be difficult to identify in view of the straindiversity of these epitopes on CS) and a region for universal MHC Class II binding, such as the invariant 21 amino-acid epitope recently identified by Sinagaglia et al. (1988) which is recognized by T-cells of individuals of most HLA-DR types.

\section{CURRENT STATUS}

Mosquirix is the brand name for RTS,S which is a scientific name given to this malaria vaccine candidate and represents its composition. The ' $R$ ' stands for the central repeat region of Plasmodium (P.) falciparum circumsporozoite protein (CSP); the ' $\mathrm{T}$ ' for the $\mathrm{T}$-cell epitopes of the CSP; and the ' $\mathrm{S}$ ' for hepatitis B surface antigen (HBsAg). These are combined in a single fusion protein ('RTS') and co-expressed in yeast cells with free HBsAg. The 'RTS' fusion protein and free ' $S$ ' protein spontaneously assemble in 'RTS,S' particles. RTS,S also contains the AS01 adjuvant system, and in scientific papers is usually referred to as 'RTS,S/AS01' (MVI, 2016).

This vaccine is intended for use in areas where malaria is regularly found, for the active immunization of children aged 6 weeks to 17 months against malaria caused by the Plasmodium falciparum parasite, and against hepatitis B. After a long period of research into malaria vaccinations, Mosquirix became the first assessed vaccine (MVI, 2016). 
Data from clinical trial showed that Mosquirix provides immunity against Plasmodium falciparum malaria in children in the 12 months following vaccination protection. The study was carried out in seven African countries (Burkina Faso, Gabon, Ghana, Kenya, Malawi, Mozambique and Tanzania). The vaccine efficacy showed a 56\% of children aged between 5-17 months and in $31 \%$ of children aged 6-12 weeks following vaccination. (European Medicine Agency, 2015). This was effective at preventing a first or only clinical malaria episode in. The efficacy of the vaccine decreased after one year. The safety profile of the vaccine was considered acceptable (Wilby et al., 2012).

From the results of studies, Mosquirix does not offer complete immunity, and the immunity it provides decreases in the longer term, despite this limitation, its efficacy outweigh the risk it is important that established protective measures, for example insecticide-treated bed nets, continue to be used in addition to the vaccine The Committee for Medicinal Products for Human Use (CHMP) considered that the benefits of immunization may be particularly important among children in endemic areas in which mortality is very high. (European Medicine Agency, 2015).

The most recent advance in RTS,S/AS01 vaccine development has been made through a modification of dose and schedule: 10/16 (62\%) volunteers given the full dose at the standard 0-1-2 month regimen were protected against CHMI 3 weeks after the last immunization; in contrast, 26/30 (86\%) volunteers were protected when the third vaccine administration occurred 6 months after the second with the dose reduced to one-fifth of the original dose. Immunological analysis to determine the mechanism for these findings showed that the titers of anti-NANP antibody were similar between the two groups but the Fx regimen may affect antibody avidity, somatic hypermutation, and isotype switching (Regules et al., 2016).

While it remains unclear whether the prolonged interval and/or the reduced dose of the final vaccine are mediating these effects, the data raise interesting questions about how this simple alteration controls antibody quality. However, it is important to note that while short-term protection was strikingly improved with the Fx regimen, only 3/7 subjects were protected following secondary CHMI 8 months later. These data suggest that durability of protection will need to be further assessed in future clinical trials and likely improved and, importantly, it remains to be determined whether this altered schedule will lead to improved protection in the field. Important new information will also emerge from clinical testing of next-generation vaccine designs. The $\mathrm{R} 21$ vaccine is composed of a single subunit (equivalent to "RTS" alone without the 4-fold excess HBsAg) (Collins, et al., 2017). The display of a higher proportion of PfCSP and less HBsAg per VLP may lead to improved anti-NANP IgG responses in comparison to RTS,S. Clinical testing is underway using Matrix-M or AS01 adjuvants.

The most extensively tested vaccine candidate for prevention of $P$. falciparum malaria is RTS,S/AS01; this vaccine directs immune responses against the major circumsporozoite protein (PfCSP) covering the surface of the infecting sporozoite. To accomplish this, RTS,S was designed as a virus-like particle (VLP) comprised of two components: 18 copies of the central repeat and the C-terminal domain of PfCSP fused to hepatitis B virus surface antigen ( $\mathrm{HBsAg}$ ) with extra HBsAg in a 1:4 ratio. RTS,S, formulated with the potent liposomal adjuvant system AS01 from GlaxoSmithKline, is the only vaccine that has demonstrated protective efficacy against clinical malaria in a Phase III clinical trial (RTS, 2015), although protection is partial, wanes over time, and may be age dependent (protection was lower in infants 6-12 weeks of age than in young children 5-17 months old). In the latter, receiving three vaccinations in a $0-1-$ 2 month schedule, the incidence of clinical malaria was reduced by $51 \%$ over the first year of follow-up postdose three [95\% CI 48\%-55\%]. Over 48 months of follow-up, efficacy was $26 \%$ [95\% CI $21 \%-31 \%$ ], and among children receiving a fourth dose at month 20 (18 months post-dose three), efficacy was 39\% [95\% CI $34 \%-43 \%]$.

A small Phase II study, which followed several hundred children who received the three-dose regimen over 7 years, suggests that there may also be a shifting or rebound in malaria incidence 5 years post-vaccination (Olotu et al., 2016). According to the World Health Organization in 2016, two safety signals (meningitis, cerebral malaria) emerged from the Phase III trial, for which the cause is unknown and they noted a confirmed risk of febrile convulsions within 7 days of vaccination in the 5-17 month age category, all of which resolved without long-term sequelae (WHO, 2016).

Following a positive opinion from European regulatory authorities in July 2015, WHO recommended largescale pilot implementations to further evaluate the feasibility of delivering four doses, the vaccine's potential for reducing childhood deaths, and to provide additional data on safety in the context of routine use. The pilot implementation program will include robust safety surveillance of these and other safety signals 
(Klein et al., 2016) that could not be adequately assessed in the Phase III trial due to very low mortality in the trial overall.

Recent progress has been made with the completion of a Phase 3 trial of the RTS,S/AS01 candidate vaccine and review by the European Medicines Agency and WHO. There is currently no commercially available malaria vaccine. Over 20 other vaccine constructs are currently being evaluated in clinical trials or are in advanced preclinical development (WHO, 2019).

Mosquirix is intended for use in areas where malaria is regularly found, for the active immunisation of children aged 6 weeks to 17 months against malaria caused by the Plasmodium falciparum parasite, and against hepatitis B. After decades of research into malaria vaccinations, Mosquirix is the first vaccine for the disease to be assessed by a regulatory agency (EMA, 2015).

Malawi became the first country to begin the use of the mosquirix vaccine as part of routine childhood immunization programs the pilot studies began on 23 April 2019, Ghana and Kenya have started large-scale vaccinations in 2019 (WHO, 2019). Mosquirix - the brand name for RTS,S - triggers the immune system to defend against the first stages of the infectious disease shortly after the malaria parasite enters the bloodstream through a mosquito bite (DW, 2019). Currently no vaccine pilot or trial studies in Nigeria, other methods of malaria elimination are in progress.

A novel subclass of highly potent anti-PfCSP antibodies, which bind a unique and conserved site of vulnerability found at the junction between the R1 cleavage site and the repeat region, has also been recently reported. These “junctional-binding" mAbs appear to be superior to comparator anti-NANP antibodies for protection in vivo as assessed against $P$. falciparum infection in humanized liverchimeric mice (Kisalu et al., 2018; Tan et al., 2018). Given that RTS,S contains NANP repeats but lacks the full junctional epitope, it will be interesting to see whether these emerging structure-function data can now be harnessed for superior immunogen design leading to more potent anti-PfCSP pAb responses (Kisalu et al., 2018).

\section{FUTURE PROSPECT}

One of the most important imperatives for future improvements to RTS,S/AS01, and all next-generation malaria vaccines, is to extend the period of protection, which will require further understanding the mechanisms of vaccine-induced efficacy. While a definitive immune mechanism remains to be determined for RTS,S/AS01, the existing data strongly suggest that high antibody concentrations against the NANP amino acid repeats are closely associated with protection, and waning of such responses is likely to be responsible for decreasing efficacy (White et al., 2015).

A direct mechanistic link between a monoclonal antibody (mAb) against the NANP epitope (isolated from a subject immunized with RTS,S (Oyen, et al., 2017) and protection will be tested soon following passive transfer and controlled human malaria infection (CHMI). RTS,S also contains the C-terminal region of PfCSP; however, the role of antibody responses to this region remains unclear as a recent study showed that a number of such mAbs, obtained from a human subject immunized with sporozoites, are not protective in a mouse model (Scally et al., 2018). In addition to antibody, CD4+ T cell responses have been suggested to have some role in protection (Kazmin et al., 2017). In some support of this, the $\mathrm{C}$ terminus of PfCSP, present in RTS,S, contains two well-defined CD4+ T cell epitopes, and a genetic analysis of the Phase III trial indicates a significant sieving effect with modestly lower efficacy for C-terminal sequence unmatched strains (Neafsey et al., 2015).

However, a definitive immune mechanism for this effect remains to be determined, and for now the major limitation of RTS,S appears to be maintaining the high antibody levels. It therefore seems critical for future PfCSP-based subunit vaccines to take into account the parameters of vaccine-induced antibody concentration decay. RTS,S is administered with the adjuvant AS01 - the leading formulation for induction of high antibody concentrations in humans. Peak polyclonal anti- NANP serum IgG responses after the final immunization averaged _ $150 \mathrm{mg} / \mathrm{mL}$ in malaria-naive adults (Kester et al., 2009), and in the Phase III trial antibody levels declined sharply with initial half-life of _40 days followed by a period of slower decline of _600 days (White et al., 2015).

Future studies will evaluate RTS,S integration into the EPI (expanded program of immunization) schedule. Private-public partnerships will be required to finance large scale phase IV vaccine trials to ensure long term success of future vaccines against malaria. For the pilot implementation studies in Ghana, Kenya, and Malawi, 750,000 children will be randomized to vaccinated and unvaccinated clusters, with four doses of RTS,S/AS01 administered in the vaccine testing group.

The impact of novel adjuvants and vaccine delivery platforms may help to provide a solution in the future, 
especially if they can be demonstrated to skew immune responses toward improved induction of long-lived plasma cells. Antibody decay parameters for an HIV envelope protein delivered with eight different clinical been studied in non-human primates (NHP) (Francica, et al., 2017), and the data demonstrate some differences in how adjuvants can influence durability. Nevertheless, improving upon the magnitude of antibody responses by adjuvants other than AS01 may be difficult to achieve.

Consequently, an alternative strategy to achieve sustained protection with lower antibody concentrations is by improving the potency or breadth of the polyclonal antibody (pAb) response. The induction of $\mathrm{pAb}$ functional at lower concentrations should result in improved vaccine efficacy during a longer segment of the IgG decay curve. Altered vaccine regimens or identification of new neutralizing epitopes on PfCSP could guide such an approach, given that RTS,S does not contain the N-terminal region and portions of the repeat region. Similarly, a more detailed understanding of the contribution made by antibody Fcmediated effector functions as well as binding parameters, such as affinity, to sporozoite blockade will be important when seeking to design vaccines that induce optimal pAb potency (Genito et al., 2017.

Important new information will also emerge from clinical testing of next-generation vaccine designs. The R21 vaccine is composed of a single subunit (equivalent to "RTS" alone without the 4-fold excess HBsAg) (Collins et al., 2017). The display of a higher proportion of PfCSP and less HBsAg per VLP may lead to improved anti-NANP IgG responses in comparison to RTS,S. Clinical testing is underway using Matrix-M or AS01 adjuvants. In an alternative approach, fulllength PfCSP containing the N-terminal non-repeat region has been manufactured for clinical testing (Genito et al., 2017), providing the potential to induce antibodies against additional epitopes (not present in RTS,S) that show anti-parasitic function in mice (Espinosa et al., 2015).

In addition to the vaccines mentioned above, there are also AMA1-Dico, GMZ2, SE36 and MSP3 which are still under clinical trials which will be available in the near future, and the data will guide further blood-stage vaccine development. It is also likely that more development efforts will be focuses on multi-antigen and multistage vaccines.

There are several considerations to accelerate future blood stage vaccine developments. As mentioned above, none of the assays/models has been proven as a surrogate of protection and no blood-stage vaccines have shown strong efficacy in a large phase II (or III) trail. Therefore, novel antigen discovery should be continued with any approach available (Amlabu et al., 2015).

\section{CONCLUSION}

Malaria vaccine developments have had great improvement over the recent years. The study that delivered irradiated Plasmodium falciparum sporozoites to vaccines by mosquito bite led to the first human malaria vaccine trail with demonstrated efficacy. The most advanced pre-erythrocytic malaria vaccine, RTS,S received a positive opinion from European regulators in 2015. RTS,S/AS01 has become the most extensively tested vaccine for prevention of Plasmodium falciparum; the vaccine directs immune responses against the major circumsporozoite protein (PfCSP) covering the surface of the infecting sporozoite. Further progress emerged to the development of Mosquirix as the first vaccine used for Malaria to be assessed by a regulatory agency. Novel adjuvants and vaccine delivery platforms may help to provide a solution in the future.

\section{ACKNOWLEDGMENT}

We remain grateful to God for seeing us through our postgraduate studies and especially in helping us to achieve this review paper. We remain indebted to our families, friends, colleagues and mentors that contributed to the success of this review. A special thanks to Dr. Adeniyi Kamoru Abdulazeez for his guidance and motivation.

\section{REFERENCES}

1. Agak G.W., Frank G., and Villard V. (2007). Rapid Identification of malaria vaccine candidates based on alphahelical coiled protein motif. PLoS one, pp2:e645.

2. Ahouidi A.D., Bei A. K., and Patel S. D. (2013). Plasmodium Falciparum merozoite surface antigen, PFRH5, elicits detectable levels of invasion-inhibiting antibodies in humans. Infectious Diseases, 208:1679-1687.

3. Amlabu E., Pandey A.K., and Reddy, K.S. (2015). Multi protein complex between the GPI-anchored CyRPA with PfRH5 and PfRipr is crucial for Plasmodiu Falciparum erythrocyte invasion. Proceedings of the National Academy of Science USA, 112 :1179-1184. 
4. Asante K. P., Adjei G., Enuameh Y. and Owusu-Agyei, S. (2016). RTS,S malaria vaccine development: progress and considerations for postapproval introduction. Archived Journal, 6: 25-32. DOI https://doi.org/10.2147/VDT.S53028.

5. Ashley E.A., Dhorda M. and Fairhurst, R.M. (2014). Spread of artemisinin resistance in Plasmodium falciparum malaria. New England Journal of Medicine, 371(5):411423.

6. Baldeviano G. C., Douglas A. D., and Lucas C.M. (2015). A PFRH5-based vaccine is efficacious against heterologous strain bloodstage Plasmodium Falciparum infection in Aotus monkeys. Cell Host Microbe, 17:130139.

7. Bang G., Kulangara, C., and Olugbile, S. (2009). Vaccine potentials of an intrinsically unstructured fragment derived from the blood stage associated $P$. falciparum protein PFF0165c. Infection and Immuninity, 77, 5701-5709.

8. Bei A. K., Membi C.D., and Rayner, J.C. (2007). Variant merozoite protein expression is associated with erythrocyte invasion invasion phenotypes in Plasmodium falciparum isolates from Tanzania. Molecular and Biochemistry Parasitology, 153, 66-71.

9. Bouharoun-Tayoun H., Attanath P., Sabchareon A., Chongsuphajaisiddhi T., and Druilhe, P. (1990). Antibodies that protect humans against Plasmodium falciparum blood stages do not on their own inhibit parasite growth and invasion in vitro, but act in cooperation with monocytes. Journal of Experimental Medicine, 172,1633-1641.

10. Chen L., Lopaticki S., and Riglar D.T. (2011). An EGF-like Protein forms a complex with PFRH5 and is required for invasion of human erythrocytes by Plasmodium Falciparum. PLoS Pathogens, 7: e1002199.

11. Clyde, D. F., Most, H, McCarthy, V. C., Vanderberg, J. P. (1973). Immunization of man against sporozite-induced falciparum malaria. American Journal of Medical Science, 266,169-177.

12. Collins K.A., Snaith R., Cottingham M.G., Gilbert S.C., and Hill, A.V.S. (2017). Enhancing protective immunity to malaria with a highly immunogenic virus-like particle vaccine. Scientific Report, 7, 46621.

13. Coursen J., Ongoiba A., and Tran T. M. (2014) Naturally acquired antibodies specific for Plasmodium falciparum reticulocyte- binding Protein homologue 5 inhibit parasite growth and predict protection from malaria. Journal of Infectious Diseases, 209, 789-798.

14. Dietz O, Kulangara, C., Luedin, S. (2012). Cell biological characterization of the malaria vaccine candidate trophozoite exported protein 1. PLoS one, 7:e46112.

15. Doolan D.L., Dobaño C., and Baird J.K. (2009). Acquired immunity to malaria. Clinical Microbiology Reviews, 22(1):13-36.

16. Douglas A. D., Williams A. R., and Worth J. J. (2011). The blood stage Malaria antigen PFRH5 is susceptible to vaccine-induciable Cross-strain neutralizing antibody. Nature communications, 2:601.

17. Espinosa D.A., Gutierrez G.M., Rojas-Lo’ pez M., Noe A.R., Shi L., Tse S.W., Sinnis, P., and Zavala F. (2015). Proteolytic cleavage of the Plasmodium falciparum circumsporozoite protein is a target of protective antibodies. Journal of Infectious Diseases, 212, 11111119.

18. Francica J.R., Zak D.E., Linde C., Siena E., Johnson C., Juraska M., Yates N.L., Gunn B., DeGregorio E. and Flynn, B.J. (2017). Innate transcriptional effects by adjuvants on the magnitude, quality, and durability of HIV envelope responses in NHPs. Blood Advances, 1, 2329-2342.

19. Genito C.J., Beck, Z., Phares, T.W., Kalle, F., Limbach, K.J., Stefaniak, M.E., Patterson, N.B., Bergmann-Leitner, E.S., Waters, N.C. and Matyas, G.R. (2017). Liposomes containing monophosphoryl lipid A and QS21 serve as an effective adjuvant for soluble circumsporozoite protein malaria vaccine FMP013. Vaccine, 35, 3865-3874.

20. Healer J. Thompson J. K. and Riglar D.T. (2013) Vaccination with conserved regions of erythrocyte - binding antigens induces neutralizing antibodies against multiple strains of Plasmodium falciparum. PLoS one, 8:e72504.

21. Kazmin D., Nakaya H.I., Lee E.K., Johnson M.J., van der Most R., van den Berg R.A., Ballou W.R., Jongert E., Wille-Reece U. and Ockenhouse, C. (2017). Systems analysis of protective immune responses to RTS,S malaria vaccination in humans. Proceedings of the National Academy of Science USA, 114, 2425-2430.

22. Kester K.E., Cummings J.F., Ofori-Anyinam O., Ockenhouse C.F., Krzych, U., Moris, P., Schwenk R., Nielsen R.A., Debebe Z. and Pinelis E. (2009). RTS,S Vaccine Evaluation 
Group Randomized, double-blind, phase 2a trial of falciparum malaria vaccines RTS,S/AS01B and RTS,S/AS02A in malarianaive adults: safety, efficacy, and immunologic associates of protection. Journal of Infectious Diseases, 200, 337-346

23. Kisalu N.K., Idris A.H., Weidle C., FloresGarcia Y., Flynn B.J., Sack B.K., Murphy, S., Schön A., Freire E., and Francica J.R. (2018). A human monoclonal antibody prevents malaria infection by targeting a new site of vulnerability on the parasite. Nature Medicine, 24, 408-416.

24. Klein S.L., Shann F., Moss W.J., Benn C.S., and Aaby P. (2016). RTS,S malaria vaccine and increased mortality in girls. MBio 7, e00514-e00516.

25. Malaria Vaccine Iniative (MVI) (2016). RTS,S (Mosquirix ${ }^{\text {TM}}$ ) Frequently Asked Questions (FAQs).

26. Neafsey D.E., Juraska M., Bedford T., Benkeser D., Valim C., Griggs A., Lievens M., Abdulla S., Adjei, S., and Agbenyega T. (2015). Genetic diversity and protective efficacy of the RTS,S/AS01 malaria vaccine. New England. Journal of Medicine, 373, 2025-2037.

27. Olotu A., Fegan G., Wambua J., Nyangweso G., Leach A., Lievens M., Kaslow D.C.,Njuguna P., Marsh K., and Bejon P. (2016). Seven-year efficacy of RTS,S/AS01 malaria vaccine among young African children. New England Journal of Medicine, 374, 2519-2529.

28. Oyen D., Torres J.L., Wille-Reece U., Ockenhouse C.F., Emerling D., Glanville J., Volkmuth W., Flores-Garcia Y., Zavala F., and Ward A.B. (2017). Structural basis for antibody recognition of the NANP repeats in Plasmodium falciparum circumsporozoite protein. Proceedings of the National Academy of Science USA, 114, E10438-E10445.

29. Oyston P., and Robinson K. (2012). The current challenges for vaccine development. Journal of Medical Microbiology, 61,889894, doi: 10.1099/jmm.0.039180-0.

30. Pandey A. K., Reddy K.S. and Singh H. (2014). Bacterially expressed full-length recombinant Plasmodium falciparum RH5 protein binds erythrocytes and elicits potent strain-transcending parasite - neutralizing antibodies. Journal of Infection and Immunity, 82,152-164.

31. Regules J.A., Cicatelli S.B., Bennett J.W., Paolino K.M., Twomey P.S., Moon J.E.,
Kathcart A.K., Hauns K.D., Komisar J.L., and Qabar A.N. (2016). Fractional third and fourth dose of RTS,S/AS01 malaria candidate vaccine: a phase 2 a controlled human malaria parasite infection and immunogenicity study. Journal of Infectious Disease, 214, 762-771.

32. Rénia L., Goh Y. S., Peng K., Mauduit M., and Snounou G. (2018). Assessing Malaria Vaccine Efficacy. Open access peer-reviewed. DOI: 10.5772/intechopen.77044

33. Sachs J., and Malaney P. (2002). The economic and social burden of malaria. Nature, 415 (6872):680-685.

34. Scally S.W., Murugan R., Bosch A., Triller G., Costa G., Mordm€uller B., Kremsner P.G., Sim B.K.L., Hoffman, S.L. and Levashina, E.A. (2018). Rare PfCSP C-terminal antibodies induced by live sporozoite vaccination are ineffective against malaria infection. Journal of Experimental Medicine, 215, 63-75.

35. Schwartz L., Brown G. V., Genton B. and Moorthy, V. S. (2012). A review of malaria vaccine clinical projects based on the WHO rainbow table. Malaria journal, 11:11.

36. Sinagaglia F., Guttinger M., and Kilgus J. (1988). A malaria T cell epitope recognized in association with most mouse and human MHC class II molecules. Nature, 336:778-80.

37. White M.T., Verity R., Griffin J.T., Asante, K.P., Owusu-Agyei S., Greenwood, B., Drakeley, C., Gesase S., Lusingu J., and Ansong, D. (2015). Immunogenicity of the RTS,S/AS01 malaria vaccine and implications for duration of vaccine efficacy: secondary analysis of data from a phase 3 randomised controlled trial. Lancet Infectious Diseases, 15, 1450-1458.

38. World Health Organization (2016). Malaria Vaccine. WHO position paper, weekly epidemiology and record. 92 (4): 33-52.

39. World Health Organization. (2016). Weekly Epidemiological Record, 91(4): 33-52.

40. World Health Organization (2018). Malaria fact sheet. Accessed 03/05/2019. 